\title{
Evolução da perda de peso entre indiví- duos da fila de espera para a cirurgia bariátrica em um ambulatório multidis- ciplinar de atenção secundária à saúde
}

\section{Evolution of weight loss among individuals in the waiting line for bariatric surgery in a multidisciplinary secondary healthcare outpatient clinic}

Cintia Satie Gushiken ${ }^{1}$, Daniela Salate Biagioni Vulcano², Ana Paula Tardivo², Irineu Rasera Jr. ${ }^{3}$, Celso Vieira de Souza Leite ${ }^{4}$, Maria Rita Marques de Oliveira ${ }^{5}$

\begin{abstract}
RESUMO
Objetivo: Caracterizar o atendimento e analisar a adesão de pacientes ao acompanhamento nutricional na fila de espera para a cirurgia bariátrica em um ambulatório multidisciplinar de atenção secundária à saúde.

Métodos: Foi realizado um estudo retrospectivo a partir de prontuários de nutrição referentes a 59 pacientes submetidos à cirurgia bariátrica no Hospital das Clínicas de Botucatu, entre 2001 e 2008 . A população estudada foi distribuída e analisada em oito grupos, conforme o tempo de acompanhamento. A análise levou em conta a evolução do peso e o número de retornos no determinado período. A comparação entre os grupos foi realizada pelo teste de Kruskal-Wallis, enquanto as correlações foram testadas pelo coeficiente de Spearman.

Resultados: Entre os 59 pacientes estudados, 42 retornaram ao serviço ao menos uma vez $(71,2 \%)$. Destes, $67,8 \%$ perderam peso na fila de espera, sendo que $27,1 \%$ dos mesmos tiveram redução maior que $10 \%$ no peso corporal. As maiores perdas de peso no pré-operatório foram encontradas nos grupos que tiveram um acompanhamento nutricional maior que seis meses. Constatou-se uma demora mediana de três meses entre os retornos, o que pode ter contribuído para a baixa adesão ao acompanhamento.

Conclusão: Os pacientes da fila de espera para a cirurgia, aqui analisados, apresentaram baixa adesão ao acompanhamento em retornos espaçados. Isso indica que para a desejada redução no peso corporal e adequado processo educativo aos candidatos à cirurgia bariátrica há que se dispor de tempo mínimo de seis meses e encontros regulares.
\end{abstract}

Palavras-chave: Obesidade Mórbida. Assistência Ambulatorial. Educação Nutricional. Cirurgia bariátrica.

1- Graduanda em Nutrição - Instituto de Biociências da UNESP de Botucatu

2- Nutricionista - Serviço Técnico de Nutrição e Dietética - Hospital das Clínicas da Faculdade de Medicina da UNESP de Botucatu.

3- Médico Cirurgião - Fundação para o Desenvolvimento Médico Hospitalar da UNESP.

4- Docente - Departamento de Cirurgia e Ortopedia da Faculdade de Medicina da UNESP de Botucatu

5- Docente - Instituto de Biociências da UNESP de Botucatu
Correspondência:

Daniela Salate Biagioni Vulcano Universidade Estadual Paulista - Faculdade de Medicina Hospital das Clinicas - Seção Técnica de Nutrição e Dietética Distrito de Rubião Junior, s/n 18618-000 Botucatu / SP dani_biagioni@yahoo.com.br

Artigo recebido em 10/09/2009 Aprovado em 26/02/2010 


\section{Introdução}

A obesidade mórbida é uma condição crônica difícil de ser tratada apenas por meio de tratamentos convencionais, como dietas e exercícios físicos. Em geral, os indivíduos com obesidade mórbida apresentam uma longa história de excesso de peso e várias tentativas frustradas de tratamentos. Foi mostrado que $95 \%$ dos pacientes que se submetem aos tratamentos convencionais para emagrecer recuperam o seu peso inicial em até dois anos do tratamento ${ }^{5}$. Neste panorama, a cirurgia bariátrica justifica-se por ser um tratamento mais duradouro e eficiente em relação à perda de peso e à resolução ou atenuação das co-morbidades ${ }^{7}$.

Os resultados favoráveis da cirurgia bariátrica aliados à expansão da prevalência da doença têm provocado aumento do número deste procedimento no mundo todo, inclusive no Brasil. Entretanto, o tratamento cirúrgico só deve ser realizado quando outros tratamentos para redução de peso (dieta, atividade física, medicamentos e mudança no comportamento) tenham sido comprovadamente mal sucedidos ${ }^{8}$.

A cirurgia é indicada para pacientes com um tempo mínimo de dois anos de evolução da obesidade e falência do tratamento convencional ${ }^{9}$. Além disso, os candidatos à cirurgia devem possuir IMC maior que $40 \mathrm{~kg} / \mathrm{m}^{2}$ ou IMC maior que $35 \mathrm{~kg} / \mathrm{m}^{2}$ associado à co-morbidades como problemas osteoarticulares, hipertensão arterial, diabetes mellitus tipo 2, doença biliar, apnéia do sono, dislipidemias ${ }^{10,11}$. É importante ainda que os candidatos ao tratamento cirúrgico não apresentem nenhuma condição adversa como risco anestésico classificado como ASA-IV (American Society of Anesthesiologists), hipertensão portal com varizes esofagogástricas, limitação intelectual significativa sem suporte familiar adequado, quadro de transtorno psiquiátrico atual não controlado, incluindo uso de álcool ou drogas ilícitas. E ainda nenhum distúrbio alimentar ou de comportamento sem controle atual ${ }^{9}$.

Os melhores resultados da cirurgia são alcançados quando os pacientes recebem atenção de uma equipe multidisciplinar composta pelo cirurgião bariátrico, endocrinologista, nutricionista, psicólogo e outras especialidades afins ${ }^{7}$.

A orientação nutricional pré-operatória tem como objetivo principal preparar o paciente para o programa de alimentação pós-operatório, promovendo hábitos alimentares saudáveis e adequados à nova condição anatômica do trato digestório. Os obesos com IMC acima de $50 \mathrm{~kg} / \mathrm{m}^{2}$ têm a recomendação de perder por volta de $10 \%$ do peso antes da operação, a fim de garantir melhor controle das co-morbidades e reduzir o risco cirúrgico e anestésico ${ }^{7}$.

Cruz $^{12}$, revisando trabalhos de outros autores, indica que o aconselhamento nutricional anterior à cirurgia tem por finalidade conscientizar o paciente quanto aos novos hábitos alimentares que lhe serão impostos, identificar possíveis riscos nutricionais, como a presença de transtornos alimentares, assim como discutir as expectativas do paciente relativas à perda de peso, a importância das recomendações nutricionais pós-operatórias para o sucesso sobre a perda de peso e a qualidade de vida. $\mathrm{O}$ aconselhamento nutricional deve levar em conta as mudanças na capacidade gástrica e as restrições dietéticas, sendo esses objetivos frequentemente buscados num intervalo de tempo relativamente próximo à cirurgia. Entretanto, discute-se a necessidade de maior aprofundamento na abordagem nutricional do paciente da fila de espera para a cirurgia, aquele que por definição fracassou em outros tratamentos.

Desse modo, o presente estudo teve como objetivo caracterizar o atendimento ambulatorial e analisar a adesão de pacientes ao acompanhamento nutricional na fila de espera para a cirurgia bariátrica em um ambulatório multidisciplinar de atenção secundária à saúde.

\section{Metodologia}

Foram analisados 59 prontuários de nutrição, selecionados entre 97 pacientes que fizeram cirurgia bariátrica pela técnica de Derivação Gástrica em Y de Roux (DGYR) com anel de contenção gástrica por laparotomia, no período de 2001 a 2008, no Hospital das Clínicas de Botucatu. Como critérios de inclusão foram aceitos indivíduos de ambos os sexos, submetidos à cirurgia bariátrica, que compareceram às consultas de nutrição no período pré-operatório. Foram excluídos do estudo pacientes que fizeram a cirurgia bariátrica por outras técnicas e/ou que não foram acompanhados pela equipe de nutrição do Serviço de Nutrição do Hospital das Clínicas de Botucatu no período pré-operatório $(\mathrm{n}=38)$.

O levantamento de informações constou da observação da rotina do ambulatório e consultas em prontuários. Foram levantadas informações sobre o fluxo dos pacientes ao serviço, forma de agendamento e tipo e protocolos de atendimento. Nos prontuários foram levantadas informações sobre sexo, idade, tempo de acompanhamento, peso, intervalo e número de retornos. 
Para fins de análise, os dados dos pacientes foram colhidos dos prontuários e separados em oito grupos de acordo com o tempo de acompanhamento nutricional antes da cirurgia (Grupo 1 - nenhum acompanhamento, Grupo 2 - acompanhados entre um e seis meses, Grupo 3 - acompanhados entre seis e 11 meses, Grupo 4 - acompanhados entre 12 e 17 meses, Grupo 5 - acompanhados entre 18 e 23 meses, Grupo 6 - acompanhados entre 24 e 29 meses, Grupo 7 acompanhados entre 30 e 35 meses, e Grupo 8 - acompanhados por mais de 36 meses). Foi também calculado o número mediano de retorno de cada grupo.

Para avaliação da evolução do peso foram considerados apenas os indivíduos que tiveram três meses ou mais de acompanhamento $(n=42)$.

O tempo de acompanhamento foi dado pela diferença de meses entre a primeira e a última consulta pré-operatória.

Os resultados foram expressos em percentual, média mais ou menos desvio padrão ou mediana e apresentados em gráficos ou tabelas. Considerando que os dados obtidos não apresentaram distribuição normal sob a curva, o teste não paramétrico de Kruskal-Wallis foi aplicado nas comparações das variáveis entre os grupos. O coeficiente de correlação de Spearman foi empregado para testar a correlação entre a perda de peso, o tempo de acompanhamento e o número de retornos. O nível de significância adotado foi de $5 \%(p d " 0,05)$.

\section{Resultados}

Quanto à caracterização do atendimento no Ambulatório de Obesidade Mórbida durante o período sob análise, o agendamento ocorreu por meio do encaminhamento por um profissional a partir da necessidade percebida quanto ao tratamento cirúrgico, ou ainda por livre demanda, quando os pacientes obesos mórbidos procuraram o serviço do ambulatório manifestando interesse em realizar a cirurgia bariátrica, ou ainda buscando tratamento convencional para emagrecimento.

O acompanhamento do obeso mórbido foi por tempo indeterminado e os retornos dependeram das vagas disponíveis no ambulatório, não havendo um protocolo específico para os casos de tratamento convencional, fila de espera para a cirurgia ou orientações para o período pré-operatório. Cada caso foi tratado particularmente, em consulta individual.
No atendimento nutricional, a avaliação antropométrica consistiu da aferição do peso e estatura para classificação do IMC conforme a Organização Mundial de Saúde de $1998^{6}$. Na primeira consulta foi seguido um protocolo de atendimento da obesidade mórbida, que reunia dados sócio-econômicos, dados do estilo de vida como atividade física, fumo e etilismo, início do ganho de peso, tratamentos anteriores e um recordatório alimentar de 24 horas (R24h). A avaliação do peso e do consumo alimentar pelo R24h repetiu-se a cada retorno do paciente.

Após a anamnese do primeiro atendimento, os pacientes foram orientados com informações gerais sobre como realizar uma alimentação saudável. Nos atendimentos seguintes, depois de calculadas as necessidades energéticas pela nutricionista, os pacientes receberam planos alimentares individualizados.

Dos pacientes estudados, $17 \%(n=10)$ eram do sexo masculino e $83 \%(n=49)$ do sexo feminino. A idade média encontrada foi de 43,8 $\pm 9,9$ anos. Na tabela 1 são apresentados os números absolutos e relativos dos indivíduos que compuseram cada grupo de análise. Verifica-se que $59 \%$ dos pacientes foram acompanhados por um período menor que um ano e que um terço dos pacientes não retornou ao serviço.

A mediana de retornos ao serviço encontra-se em torno de duas a três vezes ao ano, variando conforme o grupo (Tabela 1). Em termos globais, a mediana de emagrecimento foi correspondente a 0,00 (valores máximo 22,7 e mínimo -15,67), enquanto a mediana de acompanhamento foi de 6 meses e de retornos foi igual a 2 .

Conforme mostra a Figura 1, 67,8\% dos pacientes perderam peso, sendo que $27,1 \%$ desses tiveram redução maior que $10 \%$ do peso corporal. A figura 1 mostra ainda que 5\% dos pacientes tiveram recuperação maior que $10 \%$ do peso corporal ao longo dos atendimentos realizados no ambulatório.

Foram encontradas maiores perdas de peso no pré-operatório dos grupos que tiveram um maior tempo de acompanhamento nutricional antes da cirurgia, conforme mostra a Figura 2.

Quando o peso foi considerado pela média mensal, foram encontradas maiores perdas mensais de peso também nos grupos acompanhados por mais tempo (Figura 3).

A Figura 4 mostra a evolução do peso corporal e indica que os resultados de redução do peso começam a aparecer após o sexto mês de acompanhamento e para o grupo com 2 ou mais anos de acompanhamento. 


\section{Tabela 1}

Distribuição dos pacientes em grupos conforme o tempo de acompanhamento no ambulatório e a mediana de retornos.

\begin{tabular}{ccccc}
\hline GRUPOS & Tempo de acompanhamento & $\mathrm{N}^{\circ}$ de indivíduos & $\%$ & $\mathrm{~N}^{\circ}$ de retornos (Mediana) \\
\hline 1 & Sem acompanhamento & 17 & 29 & 0 \\
2 & $<6$ meses & 12 & 20 & 2 \\
3 & 6 a 11 meses & 6 & 10 & 2,5 \\
4 & $12-17$ meses & 5 & 9 & 4 \\
5 & $18-23$ meses & 4 & 7 & 7 \\
6 & $24-29$ meses & 5 & 9 & 4 \\
7 & $30-35$ meses & 5 & 8 & 3 \\
8 & $>36$ meses & 5 & 8 & 9 \\
\hline Total & & 59 & 100 & \\
\hline
\end{tabular}

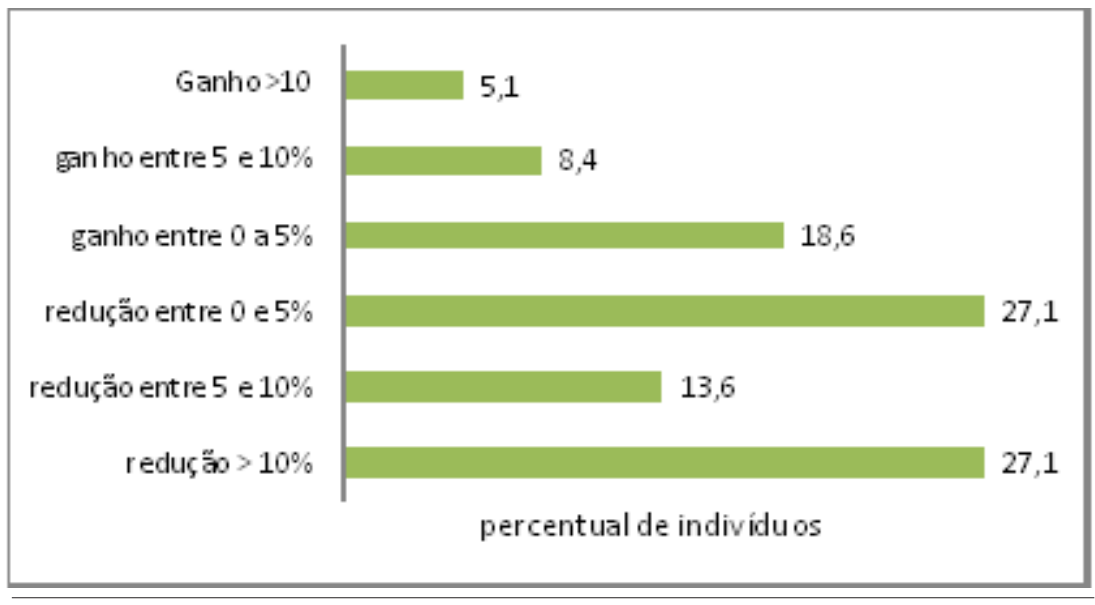

Figura 1. Distribuição dos indivíduos conforme o percentual de perda ou ganho de peso, desde o ingresso ao ambulatório até a cirurgia $(n=42)$.

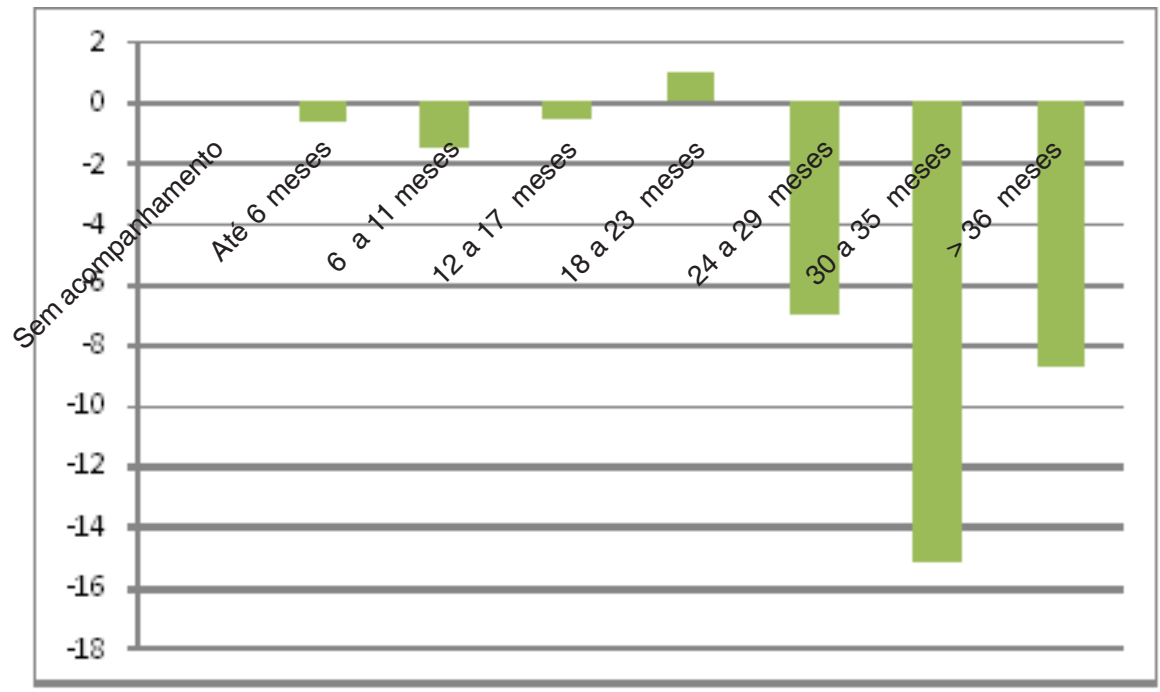

Figura 2. Valores medianos do ganho ou perda de peso acumulados entre os grupos conforme o tempo de acompanhamento $(n=59 ; p=0,051$ no teste de Kruskal Wallis, $P>0,05$ na comparação entre os grupos pelo teste de Dunn). 


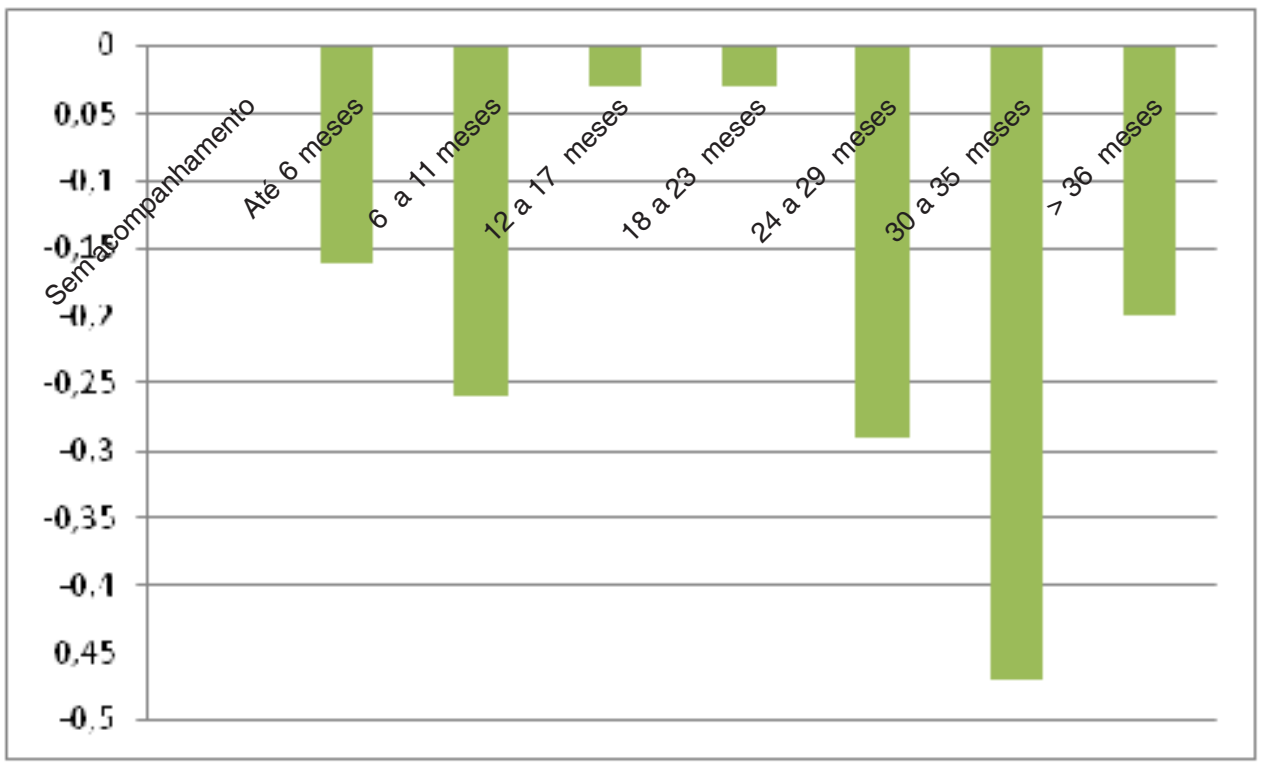

Figura 3. Valores medianos da média mensal de mudança no peso corporal (em $\mathrm{kg}$ ) dos grupos conforme o tempo de acompanhamento ( $n=59 ; p=0,009$ no teste de Kruskal Wallis, as barras marcadas com a mesma letra não diferem entre si pelo teste de Dunn ).

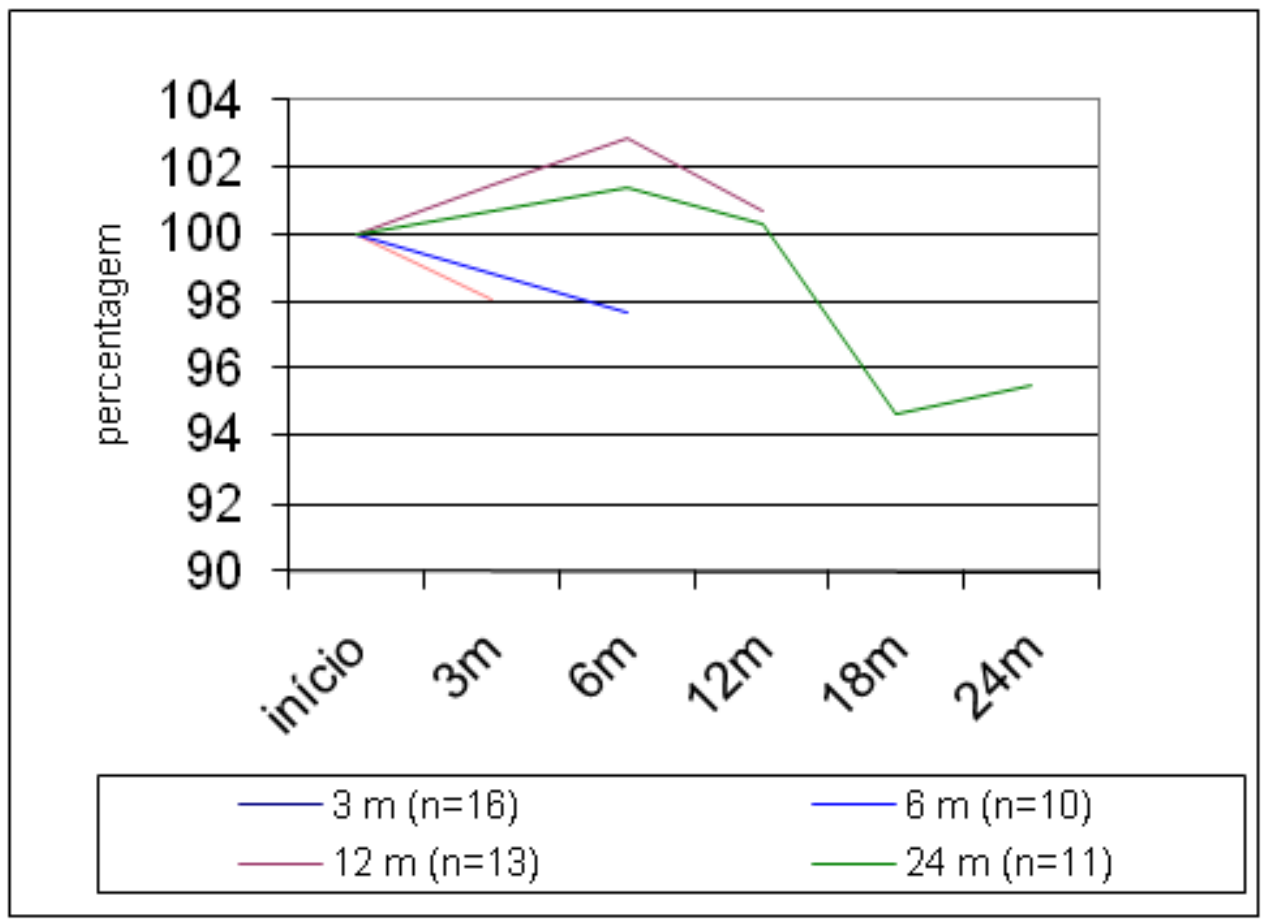

Figura 4. Evolução do peso corporal conforme o tempo de acompanhamento ( $n=42 ; p>0,05$ na comparação dos tempos entre os grupos pelo teste de Kruskal Wallis).

Quando os valores de perda de peso são comparados verifica-se que os grupos 7 e 8 , que permaneceram 30 ou mais meses em acompanhamento, foram os que apresentaram mediana de perda mensal significativamente superior aos demais grupos $(p<0,05)$.
O teste de correlação de Spearman mostrou uma fraca, porém significativa, correlação negativa entre a redução do peso corporal e o tempo de acompanhamento, mas não com o número de retornos (Figura 5). 


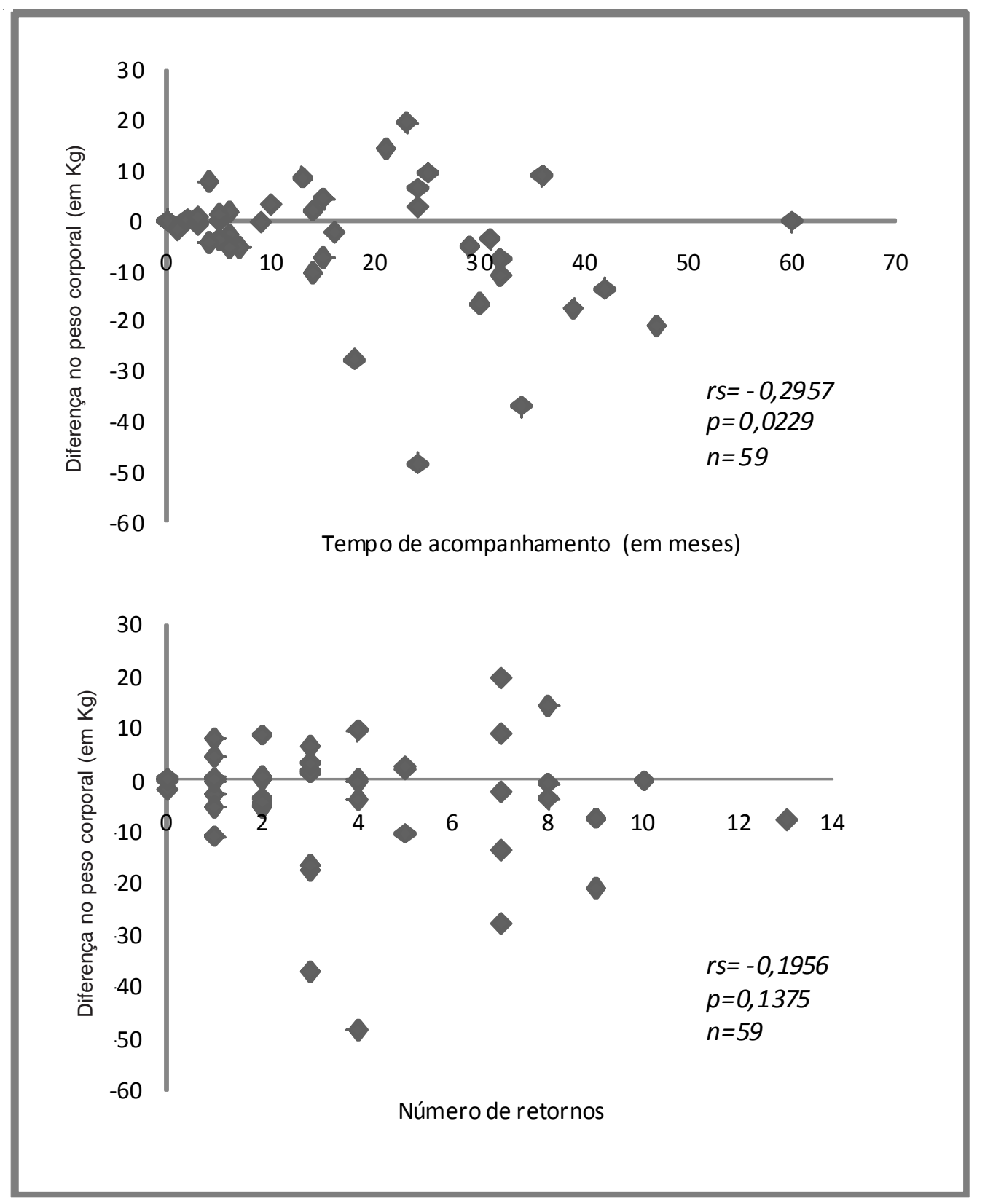

Figura 5. Correlação entre a evolução do peso corporal, o tempo de acompanhamento e o número de retornos (coeficiente de correlação de Spearman).

\section{Discussão}

O Sistema de Saúde está presenciando um aumento na demanda de cuidados voltados às doenças crônicas relacionadas com a obesidade, como diabetes tipo 2, doença coronariana, hipertensão arterial e diversos tipos de câncer ${ }^{4}$. Esse aumento reflete tam- bém numa maior demanda para a cirurgia bariátrica, fazendo com que a fila de espera pelo procedimento fique cada vez maior, tornando-se foco de preocupação dos profissionais e autoridades da saúde. Estimase que 200.000 pessoas morrem anualmente por causa das complicações da obesidade na América Latina ${ }^{4}$. 
Lottenberg $^{13}$ afirma que redução modesta de peso $(5 \%$ a $10 \%)$ resulta na melhora de diversos indicadores clínicos do paciente obeso, o que poderia diminuir o risco de mortes na fila de espera. No entanto, no presente trabalho foi encontrado que apenas 40,7\% dos pacientes perderam mais de $5 \%$ do peso corporal no período que precedeu a cirurgia, sendo que a mediana geral de redução de peso corporal foi nula. Porto et al. ${ }^{14}$, em 2002, avaliaram os resultados do acompanhamento de obesos classe III no ambulatório do hospital universitário de Salvador e encontraram que apenas $26,6 \%$ dos pacientes obtiveram perda de peso entre 5 e $10 \%$ do peso inicial num período médio de 20 meses de acompanhamento, mostrando baixa resolutividade na intervenção.

Ainda para ilustrar o quão desafiadora é a intervenção para o emagrecimento, em uma revisão sistemática ${ }^{15}$ composta por 51 estudos clínicos randomizados publicados entre 1997 e 2004 foi constatado que a restrição energética promove, em média, $5 \%$ de redução do peso corporal em seis meses; 4,6\% em 12 meses e 3,0\% em 48 meses. Isso, em indivíduos obesos, não necessariamente da classe III. Com a associação do exercício ou medicamento esses valores chegam a $10 \%$ no $6^{\circ}$ mês ${ }^{15}$. Nesta mesma revisão foi apontada uma taxa de abandono do tratamento dietético superior a $25 \%$. No presente estudo $29 \%$ dos pacientes não retornaram ao serviço depois da primeira consulta (Tabela 1). Foi verificado que o tratamento em longo prazo mantém relação com a perda de peso, porém o tratamento prolongado mostrou baixa adesão. Outros estudos indicam que a obesidade necessita de tratamento em longo prazo para que os resultados sejam satisfatórios em relação à perda e à manutenção do peso corporal ${ }^{13}$; e que o tratamento mais longo está associado com a maior perda de peso $^{16}$.

O problema do abandono ao tratamento não é exclusivo da obesidade. Em um estudo sobre a adesão ao tratamento anti-hipertensivo, Giorgi ${ }^{17}$, descreveu que a taxa de abandono ao tratamento é decrescente de acordo com o prolongamento do tempo de seguimento. DiMatteo ${ }^{18}$ relatou que $38 \%$ dos pacientes deixam de seguir um tratamento agudo recomendado (por exemplo, uso de antibióticos), $43 \%$ dos pacientes não aderem a um tratamento crônico (por exemplo, tratamento anti-hipertensivo), e $75 \%$ dos pacientes não seguem as recomendações médicas relacionadas a mudanças no estilo de vida, como restrições alimentares, abandono do fumo e outros.
O maior desejo do obeso é a rápida perda de peso, razão pela qual, adere com facilidade às novas propostas de tratamento, como às dietas radicais. A adesão a essas dietas inicialmente é grande, mas não é mantida em longo prazo, por não se inserirem no cotidiano das pessoas, criando expectativas irreais quanto à velocidade de perda de peso e à quantidade de peso perdido ${ }^{13}$. Entre os pacientes do presente estudo é possível presumir que as expectativas de rápida perda de peso já se encontravam frustradas, já que todos estavam à espera da cirurgia. Esse fato pode ter contribuído ainda mais para a falta de adesão encontrada. Outro fator que pode ter contribuído com a menor perda de peso desses pacientes é a falta de disponibilidade de vagas no serviço que proporcionasse um atendimento sistemático mais freqüente. Foi visto que no ambulatório de atendimento à obesidade mórbida, no período analisado, não se dispunha de protocolo específico para o obeso na fila de espera, assim como a equipe não estava estruturada para manter os retornos em intervalos regulares.

Além disso, a adesão do paciente a uma prescrição pode ser influenciada por diversos fatores. Martins ${ }^{19}$ identificou aspectos que podem prejudicar a aderência ao tratamento, como ambiente de atendimento inadequado (ruído, luminosidade, ventilação, equipamentos), falta de organização no ambulatório (marcação de consultas e retornos, tempo de consulta, tempo de espera), uso de prescrições padronizadas, falta de material ilustrativo e tempo limitado de consulta. Holli e Calabrese ${ }^{20}$ referem que a aderência é mais bem estabelecida se o paciente encontra o mesmo profissional em cada visita, e também, que o tempo de espera para o atendimento pode ser um fator que conduz à desistência de uma próxima visita. $\mathrm{O}$ tempo entre os retornos também pode fazer com que o paciente se sinta desestimulado e abandone o tratamento. Coyne ${ }^{21}$ cita ainda que a dificuldade de adaptar o novo regime à rotina diária, a mudança necessária no estilo de vida, a falta de acesso a alimentos apropriados ou esforços extras requeridos na preparação do alimento são condicionantes impostas ao sucesso da prescrição.

Um dos papéis do nutricionista é o de ajudar as pessoas a modificarem seus hábitos alimentares, o que deve ser feito levando em conta aspectos culturais, antropológicos, socioeconômicos e psicológicos de cada indivíduo ${ }^{22}$.

A mudança de comportamento, agregando hábitos alimentares saudáveis e a prática de atividade 
física, é mais eficiente que a utilização de drogas ${ }^{23}$. Mas, como visto nos resultados do presente estudo, as mudanças no estilo de vida para a prevenção e o tratamento de doenças crônicas caracteriza-se pela baixa adesão do paciente ${ }^{24}$.

O maior problema para o obeso é a aceitação da necessidade de mudar sua atitude frente aos alimentos, não somente com o intuito de perda de peso, mas sim, com o objetivo de manter-se saudável e, conseqüentemente, atingir o peso desejado ${ }^{13}$. No caso específico dos pacientes do presente estudo, os ajustes propostos no padrão de alimentação visavam o emagrecimento pré-cirúrgico que está associado à redução do risco cirúrgico, em especial a redução do volume abdominal e depósitos hepáticos de gordura, além de educar para a adoção dos novos hábitos alimentares, que serão impostos pela cirurgia. Entretanto, nem mesmo a eminência da cirurgia foi suficiente para promover o controle da ingestão de alimentos nesta população.

\section{Conclusão}

No presente trabalho verificou-se a baixa adesão ao tratamento de pacientes na fila de espera pela cirurgia, já que menos da metade dos pacientes permaneceram vinculados ao serviço por mais que um ano. As maiores perdas de peso foram encontradas nos grupos acompanhados por um tempo maior que seis meses. A demora entre os retornos pode ter contribuído para uma baixa adesão ao tratamento. Isso indica que para a desejada redução no peso corporal e adequado processo educativo prévios à cirurgia há que se dispor de tempo mínimo de seis meses e encontros regulares. Destes resultados se presume a necessidade de estratégias para aumento no tempo de acompanhamento do paciente que aguarda a cirurgia bariátrica e redução do tempo entre os retornos, para que ele tenha maiores condições de mudar seus hábitos e estilo de vida, indispensáveis ao sucesso da cirurgia.

\begin{abstract}
Objective: The objective of this study is to characterize the assistance and analyze patient's adherence to nutritional follow-up while in the waiting line for bariatric surgery in a multidisciplinary, secondary healthcare outpatient clinic.

Methods: This retrospective study was based on the nutritional records of 59 patients subjected to bariatric surgery at the Hospital das Clinics de Botucatu, from 2001 to 2008. The population studied was distributed into 8 groups and analyzed according to follow-up duration. The analysis took into account the evolution of body weight and the number of follow-up visits in a given period. The Kruskal-Wallis test was used to compare data between groups while Spearman's coefficient was used to test correlations.

Results: Among the 59 studied patients, $42(71.2 \%)$ returned to the clinic at least once in six months. Of these, $67.8 \%$ lost weight while in the waiting line and $27.1 \%$ of them lost more than $10 \%$ of their body weight. The greatest weight losses in the preoperative period were found in the groups that had more than six months of nutrition follow-up. There was a mean delay of three months between return visits, which could have contributed to the low adherence to follow-up.

Conclusion: The patients in the waiting line for surgery analyzed in this study presented low adherence to the long-spaced return visits. The greatest weight losses were observed in groups that had been followed for more than six months. This shows that, to achieve the desired weight loss and an adequate education process before surgery, at least six months of follow-up and regular visits are necessary.
\end{abstract}

Keywords: Obesity, Morbid. Ambulatory Care. Nutrition Education. Bariatric Surgery.

\section{Referências bibliográficas}

1. Instituto Brasileiro de Geografia e Estatística. Pesquisa de Orçamentos Familiares - POF 2002-2003 [homepage da Internet]. Brasília: IBGE; 2009 [acesso 23 abr 2009]. Disponível em: www.ibge.gov.br/home/estatistica/populacao/ condicaodevida/pof/ 2002aquisicao/default.shtm.
2. Blumenkrantz M. Obesity: the world's metabolic disorder [monografia da Internet]. Berverly Hills; 1997 [acesso 20 mar 2008]. Disponível em: http://www.quantumhcp.com, obesity.htm.

3. Cabrera M, Jacob FW. Obesidade em idosos: prevalência, distribuição e associação com hábitos e co-morbidades. Arq Bras Endocrinol Metab. 2001;45(5):494-501. 
4. Coutinho W. Consenso latino americano de obesidade. Arq Bras Endocrinol Metab. 1999;43(1):21-67.

5. Segal A, Fandiño J. Indicações e contra-indicações para realização das operações bariátricas. Rev Bras Psiquiatr. 2002;24 Supl 3:68-72.

6. World Health Organization. Obesity: preventing and managing the global epidemic. report of WHO consultation on obesity. Geneva: WHO; 1998.

7. Pereira MAG, Arasaki CH, Grande JC, Lourenço LG. Terapia nutricional na cirurgia bariátrica. In: Silva SMCS, Mura JP. Tratado de alimentação, nutrição e dietoterapia. São Paulo: Roca; 2007. p.711-23.

8. Cochrane [database on the Internet]. Técnicas de tratamento cirúrgico da obesidade mórbida: switch duodenal/ escopinaro. São Paulo: Centro Cochrane do Brasil; 2006. [acesso 25 mar 2009]. Disponível em: http://portal.saude. gov.br/portal/arquivos/pdf/switch_duodenal_escopinario_ para_obesidade_morbida_txt.pdf.

9. Sociedade Brasileira de Cirurgia Bariátrica e Metabólica [homepage on the Internet]. Consenso Brasileiro Multissocietário em Cirurgia Bariátrica. São Paulo; 2006. [Acesso 27 abr 2009]. Disponível em: http://www.sbcbm. org.br/ materias. php?cod=19.

10. National Health Institute. Consensus Development Conference Panel. NIH Conference: gastrointestinal surgery for severe obesity. Ann Intern Med. 1991;115(12): 956-61.

11. Mechanick JI, Kushner RF, Sugerman HJ, Gonzales-Campoy JM, Collazo-Clavell ML, Guven S, et al. American association of clinical endocrinologists, the obesity society, and american society of metabolic \& bariatric surgery medical guidelines for clinical practice for the perioperative nutritional, metabolic, and nonsurgical support of the bariatric surgery patient. AACE/TOS/ASMBS bariatric surgery guidelines. Surg Obes Relat Dis. 2008; 4 Supl 5: S109-S84.

12. Cruz MRR, Morimoto IMI. Intervenção nutricional no tratamento cirúrgico da obesidade mórbida: resultados de um protocolo diferenciado. Rev Nutr. 2004;17(2):263-72.

13. Lottenberg AMP. Tratamento dietético da obesidade. Einstein. 2006; 4 Supl 1:S23-S28.
14. Porto MCV, Brito IC, Calfa ADF, Amoras M, Villela NB, Araújo LMB. Perfil do obeso classe III do ambulatório de obesidade de um hospital universitário de Salvador, Bahia. Arq Bras Endocrinol Metab. 2002; 46(6):668-73.

15. Almeida JC, Rodrigues TC, Silva FM, Azevedo MJ. Revisão sistemática de dietas de emagrecimento: papel dos componentes dietéticos. Bahia. Arq Bras Endocrinol Metab. 2009; 53(5):673-87.

16. Perri MG, Nezu AM, Patti ET, McCann KL. Effect of length of treatment on weight loss. J Consult Clin Psychol. 1989; 57(3):450-2.

17. Giorgi DMA. Estudo sobre algumas variáveis que influenciam a aderência ao tratamento em hipertensão arterial [tese]. São Paulo (SP): Faculdade de Medicina, Universidade de São Paulo; 1989.

18. DiMatteo MR. Enhancing patient adherence to medical recommendations. J Am Med Assoc. 1994;271(1):79-83.

19. Martins C. Aconselhamento nutricional. In: Cuppari L. Guia de nutrição: nutrição clínica no adulto. São Paulo (SP): Manole; 2002. p.111-27.

20. Holli B, Calabrese R. Communication and education skills: the dietitian guide. $2^{\text {nd }}$ ed. Pensylvania: Lea \& Febiger; 1991. p.118.

21. Coyne T, Olson M, Bradham K, Garcon M, Gregory P, Scerch L. Dietary satisfaction correlated with adherence in modification of diet in renal disease study. J Am Diet Assoc. 1995;95(11):1301-6.

22. Baldwin T, Falciglia G. Application of cognitive behavioral theories to dietary change in clients. J Am Diet Assoc. 1995;95(11):1315-17.

23. Knowler WC, Barrett-Connor E, Fowler SE, Hamman RF, Lanchin JM, Walker EA, et al. Diabetes Prevention Program Research Group. Reduction in the incidence of type 2 diabetes with lifestyle intervention or metformin. $\mathrm{N}$ Engl $\mathrm{J}$ Med. 2002;346(6):393-403.

24. Assis MAA, Nahas MV. Aspectos motivacionais em programas de mudança de comportamento alimentar. Rev Nutr. 1999;12(1):33-41. 